\title{
Collaboration as Collage
}

When artists labor together for a common purpose, the results can be transformative. Collaboration, as a creative process, engages multiple sourcing perspectives; it diversifies problem-solving skills, enhances the range of visual information used, and challenges the emotional like/dislike attachments to one's own artwork. To understand the educational benefits of collaboration, we can examine the collage process and apply the same principles.

Negation is the first and probably most important process within a collective strategy; it allows the artists to collage information over other pieces of material. This means that when two artists work together, they can layer a diverse range of ideas, forms, processes, and meanings over and over again. The constant running dialogue two or more people have when working together is the bond or the adhesive. They need to communicate with each other for the work to materialize. Discussion leads to production. Edges remind me of how time is used in a cooperative project. A collage's edges can be clean and hard, torn, fuzzy, soft, or seamless. We can imagine artists working shoulder to shoulder, or passing the work back and forth for different intervals of time. The edge is the physical passing of object and idea from one person to another.

Materiality contrasts are very common in collage because of its cut and paste history. Again, you can use a multitude of different material ideas in one piece. This echoes the different technical strengths, material sensibilities, and conceptual interests each artist brings to the picture. This often allows the most unlikely artists to succeed together. Collage offers the fluid ability to crop, arrange, skew, and distort with frenzied speeds. This mimics the shared understanding to eventually own certain territory on the picture plane. Artists can usually identify their marks and nothing is ever completely integrated, at least from the artist's perspective.

The studio classroom and the laboratory are the same; art students are constantly experimenting, cataloging, and surveying their progress with problem solving intentions. I have used collaboration techniques for over ten years in the classroom and have seen many results, both successes and failures. Failure is of course a positive force in art making. It allows us to take great risks and not be timid when building language. A university art studio 
inherently has collaboration built right into its space; students as well as teachers gather to share ideas and to generate work as a collective. Structured art courses serve to enhance the collage nature of a studio class; they resolve, develop, critique, and mature the work as one cohort that is responsible for each other's learning outcomes. Human relationships form and collaboration becomes a vehicle for healthy communication.

The exhibition of their artwork is the final collaboration. The installation of a project becomes an artwork in itself, which requires a team of people to complete. Curators, community based artists, teachers, and students work together during this process. Considerations include the selection of artworks around themes and narratives, spatial placements, lighting, text and image relationships, installation mechanics, public events, tours, marketing, and numerous other items. Throughout this process, community is built, culture is fostered, and the public invitation is the final crucial step in the cycle of a creative community.

Shawn Serfas 